\title{
COMPORTAMENTO DA MADEIRA DE Tectona grandis FRENTE AO INTEMPERISMO
}

\author{
Robert Rossi Silva de Mesquita ${ }^{1 *}$, Joaquim Carlos Gonçalez ${ }^{2}$, Marcella Hermida de Paula ${ }^{1}$ \\ ${ }^{1 *}$ Universidade de Brasília, Programa de Pós Graduação em Ciências Florestais, Brasília, Distrito Federal, Brasil - \\ robertflorestal@gmail.com; marcellahermida@hotmail.com \\ ${ }^{2}$ Universidade de Brasília, Departamento de Engenharia Florestal, Brasília, Distrito Federal, Brasil - goncalez@unb.br
}

Recebido para publicação: 13/11/2014 - Aceito para publicação: 15/02/2017

\begin{abstract}
Resumo
A qualidade da madeira pode ser alterada por intempéries que podem diminuir sua vida útil, bem como alterar a sua cor, que é um fator preponderante no mercado madeireiro, muitas vezes determinando seu preço final, principalmente quando utilizada em ambientes internos. A Teca (Tectona grandis Linn. F.) é uma madeira comercialmente valiosa, possui propriedades físico-mecânicas e características qualitativas como a cor, o desenho e a densidade desejáveis em produtos que requerem um bom acabamento. O objetivo deste estudo foi avaliar o processo de intemperismo artificial da madeira de Teca submetida a 5 ciclos de irradiação UVA de $1,55 \mathrm{~W} / \mathrm{m}^{2}$ a $340 \mathrm{~nm}$ de comprimento de onda, conforme a norma ASTM G154. O sistema CIE L*a*b* de 1976 mostrou as modificações das cores. A mudança da cor natural da madeira, indicativo do processo de fotodegradação, foi monitorada por espectrocolorimetria. Amostras da espécie não sofreram alteração de cor frente ao intemperismo, permanecendo na cor marrom oliva, tornando-a uma opção frente a outras espécies madeireiras de uso em escala industrial, principalmente naqueles onde consegue-se agregar mais valor.

Palavras-chave: Espectrocolorimetria; fotodegradação; CIE L*a*b*; teca; cor.
\end{abstract}

\begin{abstract}
Behavior of tectona grandis front of weathering. The quality of the wood might be affected by weathering, decreasing its life span, as well its color, which constitutes in a preponderant factor for the wood market, sometimes, determining its final price, especially when the material is used indoors. The Tectona grandis Linn. F. is a commercially valuable wood, due its physical and mechanical proprieties, and qualitative characteristics such as color, design and density are suitable for products that require a good finishing. This research aimed to evaluate the artificial weathering process on the Tectona grandis Linn. F. wood, submitting it to 5 UVA radiation cycles of $1,55 \mathrm{~W} / \mathrm{m}^{2}$ at $340 \mathrm{~nm}$ of wave-length, in accordance to the ASTM G154 norm. The CIE $\mathrm{L}^{*} \mathrm{a}^{*} \mathrm{~b}^{*}$ system from 1976 showed the changes on the colors. The modification of the natural color of the wood, an indicator of the photo-bleaching process, was monitored through spectrocolorimetry. The weathering did not change the color of the samples evaluated, therefore, its color remained olive-brown, making the species a viable option for industrial use, mostly on higher priced products.
\end{abstract}

Keywords: Spectrocolorimetry; photo-bleaching; CIE L*a*b*; Tectona grandis Linn. F; color.

\section{INTRODUÇÃO}

A principal atividade do setor florestal é, sem dúvidas, a produção madeireira, a qual é utilizada em vários setores da economia, com finalidade moveleira, produção de celulose e papel, construção civil, produção de energia, entre outros.

Quando a madeira é submetida a condições ambientais externas ou internas, pode sofrer processos químicos, físicos e mecânicos, alterando suas características (NZOKOU; KAMDEM, 2002). As características tecnológicas da madeira são as que determinam seu uso mais apropriado. Entretanto, estas características podem ser alteradas pelo processo de deterioração ocasionado pelo tempo, atrelado a ação de vários outros fatores. A madeira exposta à radiação solar está sujeita à deterioração superficial, ou chamada fotodegradação, diminuindo o seu tempo útil de vida. Primeiramente, ocorre uma mudança de cor e depois uma perda de suas propriedades de uma forma geral (GONÇALEZ et al., 2010).

Quando exposta a fatores como, umidade, raios ultravioletas, temperatura, agentes químicos e biológicos, a madeira sofre o que é chamado de intemperismo. (WILLIANS, 2005).

Apesar da ação do intemperismo ser limitada à superfície da madeira, constitui um problema para os seus usuários, pois afeta o desempenho dos produtos aplicados sobre a mesma. Por exemplo, as resinas que contêm compostos ditos resistentes a radiação UV, possuem tempo de vida limitado, exigem manutenção frequente, o que

FLORESTA, Curitiba, PR, v. 47, n. 1, p. 29 - 35, jan. / mar. 2017.

Mesquita, R. R. S. de et al.

ISSN eletrônico 1982-4688

DOI: $10.5380 /$ rf.v47i1.38562 
inclui a sua remoção e preparação de nova superfície (FEIST; HON, 1984; MARTINS et al., 2011).

Além disso, os produtos semitransparentes permitem que a radiação atinja a superfície da madeira, deteriorando-a antes mesmo do revestimento (PANDEY; PITMAN, 2002; SILVA et al, 2007). A peça de madeira quando exposta à luz absorve intensamente a radiação solar e sofre degradação fotoquímica, fenômeno artificial, devido à ação dos raios ultravioleta, que comprometem o seu aspecto geral, tornando-a descorada ou acinzentada, amarelada ou escurecida, dependendo da influência da composição da madeira, principalmente dos extrativos (AYADI et al, 2003; COSTA et al, 2011).

Pelo panorama químico, os extrativos são compostos cromóforos responsáveis pelo odor e pela cor da madeira. Como são absorventes da radiação solar causam alteração de cor na madeira exposta externamente (PANDEY, 2005).

Barros (2014) menciona que cor é uma das características mais importantes para a identificação, classificação e indicação de uso de espécies de madeira, principalmente quando associada aos aspectos de textura e desenho em usos finais com maior valor econômico.

Mori et al. (2005) afirma que a cor vem sendo considerada também como um indicador de qualidade da madeira já que é uma característica tão importante quanto as propriedades físicas e mecânicas. Por esta razão, a análise da cor deve ser incorporada ao planejamento visando a caracterização tecnológica da madeira a fim de atender aos usos mais nobres desse material.

A teca é uma madeira de alto valor comercial, segundo da Costa e de Resende (2007), a madeira da teca alcança bons preços e, compete, no momento em igualdade de situação com madeiras consideradas nobres mundialmente, em especial comparada ao mogno. Trata-se de uma das espécies mais procuradas e valorizadas no mercado internacional de madeiras por sua alta durabilidade, boa estabilidade dimensional, resistência, peso e pelas qualidades estéticas de sua madeira (MOYA; PÉREZ, 2007; MIRANDA et al., 2011; TONINI et al, 2010).

O objetivo desse trabalho foi o de verificar o comportamento da cor da madeira de teca (Tectona grandis) frente ao intemperismo artificial, visando um melhor conhecimento desta espécie, para a sua maximização de uso na indústria.

\section{MATERIAL E MÉTODOS}

\section{Origem da madeira e preparo dos corpos de prova}

A madeira de Tectona grandis (Teca) utilizada neste estudo foi procedente do estado do Mato Grosso (MT), mais precisamente da empresa Floresteca. O plantio estava com 10 anos de idade. Para a realização deste trabalho foram confeccionadas vinte amostras constituídas de cerne, com dimensões de $80 \times 30 \times 5 \mathrm{~mm}$ (comprimento x largura x espessura), provenientes de 3 pranchas, medindo $2,5 \mathrm{~cm}$ x $10 \mathrm{~cm}$ x $150 \mathrm{~cm}$. Os ensaios de intemperismo e colorimetria foram realizados no Laboratório de Tecnologia da Madeira do Departamento de Engenharia Florestal da Universidade de Brasília.

\section{Ensaio de intemperismo}

Para este ensaio as amostras foram lixadas com lixa para madeira de grão 120 com o intuito de se retirar as impurezas superficiais e marcas ocasionadas pela lâmina de serra durante a confecção dos mesmos, além dos efeitos já provocados pelas radiações ultravioletas. As amostras foram acondicionadas imediatamente em uma caixa de papelão com tampa para proteção contra a luz. O ensaio de intemperismo acelerado por meio de irradiação ultravioleta artificial foi feito utilizando a metodologia adotada por Gonçalez e Zerbini (2010).

As amostras foram introduzidas em uma câmara de envelhecimento acelerado marca QUV/Spray, composta por placas suportes metálicas, deixando a face tangencial da madeira exposta ao intemperismo artificial. Este equipamento possui controle automático de temperatura e umidade relativa utilizando lâmpadas fluorescentes UVA com irradiância de $1,55 \mathrm{~W} / \mathrm{m}^{2} / \mathrm{nm}$ com comprimento de onda de $340 \mathrm{~nm}$, conforme a norma ASTM G154 (2006), onde permaneceram por 5 ciclos contabilizando 288 horas. Pela norma, a programação utilizada foi a de ciclo 7 , sendo a mais severa, no qual as condições de exposição das amostras foram de 8 horas de irradiação UV a $60( \pm 3)^{\circ} \mathrm{C}$ de temperatura; 0,25 horas de exposição ao spray de água (sem luz) e condensação de 3,75 horas a $50( \pm 3)^{\circ} \mathrm{C}$ de temperatura. $\mathrm{O}$ controle do experimento de intemperismo na madeira foi feito pela colorimetria (sistema CIELAB). Inicialmente e após cada ciclo as amostras de madeira foram retiradas da câmara para realização da medição colorimétrica. Os ciclos de intemperismo foram acumulativos na seguinte ordem: 0-12-24-96-288 horas, totalizando 5 tratamentos.

\section{Colorimetria da madeira}

Para avaliação da mudança de cor antes (testemunha) e em cada ciclo de horas de exposição do intemperismo, foi utilizado um espectrofotômetro ColorEye XTH marca X-Rite, nas condições: iluminante D65 e ângulo de $10^{\circ} \mathrm{em}$ temperatura ambiente, acoplado a um microcomputador, utilizando o software Icolor rite, 
tomando-se medidas dos parâmetros colorimétricos na face tangencial da madeira. As leituras foram feitas no sentido das fibras, totalizando 15 medições por amostra em cada ciclo de intemperismo e utilizando por cima de cada amostra um mesmo molde de papel com 15 furos equidistantes nos quais foi posicionado o espectrofotômetro para a obtenção dos dados nos mesmos pontos em todos os tratamentos. Em cada ponto de medição o espectrofotômetro utilizado fornece automaticamente os cinco parâmetros colorimétricos $\left(\mathrm{L}^{*}, \mathrm{a}^{*}, \mathrm{~b}^{*}\right.$, $\mathrm{C}$ e $\mathrm{h}^{*}$ ) de acordo com a norma CIELAB. Obtidas todas as medições, foi feito a média dos 15 pontos por amostra e posteriormente a média total dos pontos para cada tempo de intemperismo artificial. Com a média total dos parâmetros colorimétricos $\left(\mathrm{L}^{*}, \mathrm{a}^{*}, \mathrm{~b}^{*}, \mathrm{C}\right.$ e $\left.\mathrm{h}^{*}\right)$ por período de tempo, foi possível utilizar uma tabela de cores sugerida por Camargos e Gonçalez (2001), para caracterizar a cor da madeira de teca em cada tratamento. Para determinar as variações de cores dos parâmetros colorimétricos entre os tratamentos, utilizou-se a Equação 1 de acordo com a norma ASTM D 2244 (2009):

$$
\Delta \mathrm{E}=\sqrt{\Delta \mathrm{L}^{2}+\Delta \mathrm{a}^{2}+\Delta \mathrm{b}^{2}} \quad \text { (Equação 1) }
$$

em que: $\Delta \mathrm{E}=$ variação da cor entre o tratamento e a testemunha; $\Delta \mathrm{L}^{*}=$ variação da luminosidade; $\Delta \mathrm{a}=$ variação da coordenada $a^{*}$ (parâmetro colorimétrico posicionado no eixo de cores vermelho-verde; $\Delta \mathrm{b}=$ variação da coordenada $b^{*}$ (parâmetro colorimétrico posicionado no eixo de cores amarelo-azul).

Para classificar a variação da cor foi usada a tabela 1 elaborada por Hikita et al., (2001) baseada em níveis de percepção a olho nú.

Tabela 1. Classificação da variação total da cor $\left(\Delta \mathrm{E}^{*}\right)$ de madeiras (Hikita et al., 2001).

Table 1. Rating of the total color variation $(\Delta \mathrm{E} *)$ of timbers (Hikita et al.,2001).

\begin{tabular}{c|l}
\hline Variação da cor $(\boldsymbol{\Delta E})$ & Classificação \\
\hline $0,0-0,5$ & Desprezível \\
$0,5-1,5$ & Ligeiramente perceptível \\
$1,5-3,0$ & Notável \\
$3,0-6,0$ & Apreciável \\
$6,0-12,0$ & Muito apreciável \\
\hline
\end{tabular}

\section{Análise dos resultados}

Os dados foram analisados estatisticamente, utilizando Análise de Variância (ANOVA) em ensaio fatorial e teste de agrupamento de médias de Scott-Knott em 1\% de probabilidade de erro por meio do programa R: Pacote Experimental Design.

\section{RESULTADOS E DISCUSSÃO}

Os valores médios dos parâmetros colorimétricos da madeira de Teca perante os ciclos de exposição de intemperismo são apresentados na tabela 2.

Tabela 2. Valores médios dos parâmetros colorimétricos da madeira de Teca.

Table 2. Average values of the colorimetric parameters of the Teak.

\begin{tabular}{cccccc}
\hline $\begin{array}{l}\text { Tempo de } \\
\text { irradiação }(\mathbf{h})\end{array}$ & $\mathbf{L}^{*}$ & $\mathbf{a}^{*}$ & $\mathbf{b}^{*}$ & $\mathbf{C}$ & $\mathbf{h}^{*}$ \\
\hline 0 & $56,97 \mathrm{a}$ & $10,27 \mathrm{a}$ & $25,76 \mathrm{a}$ & $27,75 \mathrm{a}$ & $68,26 \mathrm{a}$ \\
12 & $54,12 \mathrm{~b}$ & $10,99 \mathrm{~b}$ & $28,71 \mathrm{~b}$ & $30,76 \mathrm{~b}$ & $69,12 \mathrm{a}$ \\
24 & $54,60 \mathrm{~b}$ & $11,49 \mathrm{~b}$ & $29,28 \mathrm{~b}$ & $31,48 \mathrm{~b}$ & $68,64 \mathrm{a}$ \\
96 & $55,13 \mathrm{~b}$ & $11,13 \mathrm{~b}$ & $28,12 \mathrm{~b}$ & $30,26 \mathrm{~b}$ & $68,45 \mathrm{a}$ \\
288 & $60,19 \mathrm{a}$ & $9,31 \mathrm{a}$ & $24,00 \mathrm{a}$ & $25,75 \mathrm{a}$ & $68,85 \mathrm{a}$ \\
\hline
\end{tabular}

*: Médias seguidas de uma mesma letra em uma mesma coluna não diferem entre si de acordo com o teste de Scott-Knott significativo $1 \%$ de probabilidade de erro $(* *)=$ Cor da madeira, segundo classificação proposta por Camargos e Gonçalez (2001).

Tanto a coordenada $a^{*}$, como a b* tiveram participação marcantes na formação da cor da madeira de teca. Analisando as coordenadas, verifica-se que a b* (coloração amarela) apresentou valores mais elevados quando comparados à coordenada a* (coloração vermelha), podendo se inferir que houve predominância da tonalidade amarela na formação da cor da madeira de teca, o que caracterizou a cor marrom oliva da espécie $\left(L^{*}=56,97 ; a^{*}=10,27 ; b^{*}=25,76 ; C=27,75\right.$ e $\left.h^{*}=68,26\right)$. Observa-se que nas primeiras 96 horas, apesar da madeira ter escurecido no primeiro momento ( $\mathrm{L}^{*}$ diminuiu), as coordenadas $\mathrm{a}^{*} \mathrm{e} \mathrm{b}^{*}$ aumentaram, em relação à

FLORESTA, Curitiba, PR, v. 47, n. 1, p. 29 - 35, jan. / mar. 2017.

Mesquita, R. R. S. de et al.

ISSN eletrônico 1982-4688

DOI: $10.5380 /$ rf.v47i1.38562 
testemunha, havendo uma compensação entre as pigmentações amarela e vermelha na cor final da madeira, não alterando a sua cor quando comparada com a inicial, permanecendo a cor marrom-oliva (Tabela 3), comportamento semelhante é observado para o parâmetro de saturação da cor da madeira (C) influenciado pelos parâmetros $\mathrm{a}^{*} \mathrm{e} \mathrm{b}^{*}$. É possível que a composição química dos extrativos da espécie tenha influência marcante durante este período de intemperismo, contribuindo para a estabilização de sua cor. Ao final de 288 horas de intemperismo, a madeira de teca veio a clarear e seus parâmetros colorimétricos resultaram em valores próximos ao obtido antes do ensaio.

Analisando-se ainda a tabela 2, observa-se que inicialmente, os ciclos de exposição provocaram redução na luminosidade ou escurecimento ( $\mathrm{L}^{*}$ ) das amostras. Observa-se o comportamento inverso, após o período de 24 horas de exposição a irradiação. A luminosidade começa a aumentar evidenciando o início do processo de clareamento (Figura 1). O escurecimento inicial é atribuído à formação de compostos quinoidais em função, sobretudo, da degradação da lignina pela radiação UV na presença de oxigênio (Hon, 2001). Esses compostos são lixiviados pela água, deixando a madeira mais áspera e esbranquiçada, por causa do aumento do teor de celulose que, consequentemente, causa aumento da luminosidade, este fenômeno pode explicar a significância da variação de L* no ciclo de 288 horas. Em estudo similar, SILVA et al. (2007) relata este fenômeno.

A tabela 3 apresenta os dados de variação total da cor $(\Delta \mathrm{E})$ para madeira de Teca para cada tempo de exposição classificando-se a cor de acordo a tabela proposta por Camargos e Gonçalez (2001).

Tabela 3. Variação total da cor $(\Delta \mathrm{E})$ para madeira de Teca.

Table 3. Total color variation $(\Delta \mathrm{E})$ of the Teak.

\begin{tabular}{cccc}
\hline Tempo de irradiação $(\mathbf{h})$ & $\Delta \mathbf{E}$ & Classificação $(*)$ & Nome da cor $(* *)$ \\
\hline 0 & - & - & Marrom-oliva \\
12 & 4,16 & Apreciável & Marrom-oliva \\
24 & 4,41 & Apreciável & Marrom-oliva \\
96 & 3,11 & Apreciável & Marrom-oliva \\
288 & 3,79 & Apreciável & Marrom-oliva \\
\hline$(*)$ = Classificação da variação da cor proposta por Hikita et al., (2001) baseada em níveis de percepção. $(* *)$ = Cor da madeira, segundo
\end{tabular}

classificação proposta por Camargos e Gonçalez (2001).

Observa-se na tabela 3, que apesar da percepção de variação da cor a olho nú na madeira de teca ser classificada como apreciável em todos os períodos de intemperismo, de acordo com a classificação proposta por Camargo e Gonçalez (2001), a cor da madeira ainda permanece classificada como Marrom-Oliva em todos os períodos.

A figura 1 ilustra o comportamento da Teca, evidenciado as duas fases (escurecimento e clareamento), pois ocorre a inversão na direção da curva.

\section{$\mathbf{L}^{*}$}

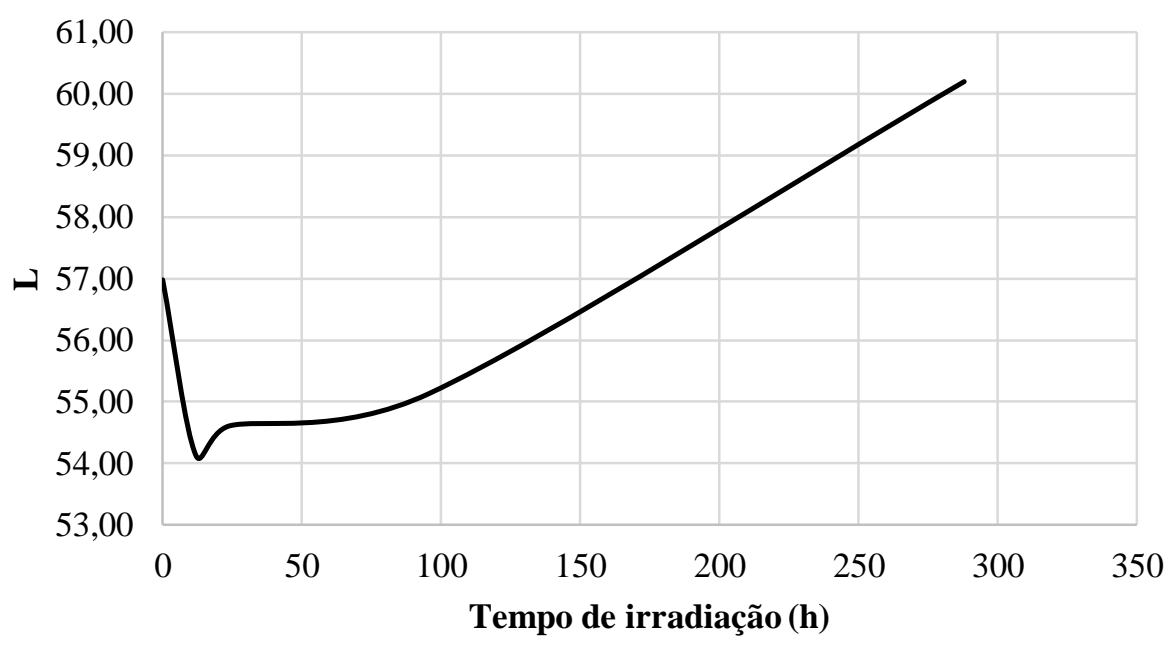

Figura 1. Curva de variação de luminosidade $\left(\mathrm{L}^{*}\right)$ da madeira de Teca provocada pelo intemperismo artificial. Figure 1. Variation curve of brightness $\left(\mathrm{L}^{*}\right)$ of Teak wood caused by artificial weathering. 
A variação total de cor $\left(\Delta \mathrm{E}^{*}\right)$, leva em consideração as diferenças das coordenadas cromáticas e de luminosidade, sendo possível obter uma visão mais ampla sobre o desempenho da madeira estudada sob o intemperismo. A figura 2 ilustra o comportamento da madeira de Teca perante ao intemperismo.

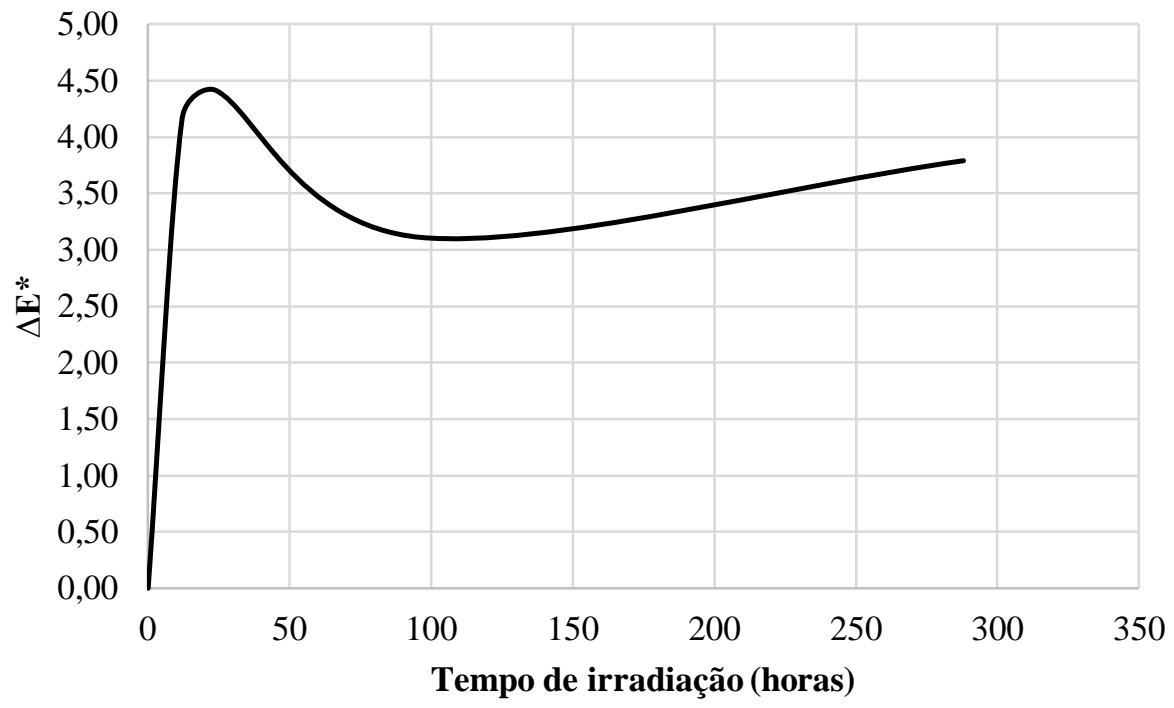

Figura 2. Curva de variação total de cor $\Delta \mathrm{E}^{*}$ causa da pelo intemperismo da madeira de Teca. Figure 2. Curve of color variation $\Delta \mathrm{E} *$ caused by artificial weathering of the Teak wood.

Apesar da classificação sugerida por Hikita et al. (2001), tabela 1, classificar a variação total da cor $(\Delta \mathrm{E})$ como apreciável para todos os tempos de intemperismo, o teste estatístico entre os parâmetros colorimétricos não mostra diferenças estatísticas, corroborando na explicação da manutenção final da cor. $\mathrm{O}$ comportamento da reflectância (Figura 3) corrobora as constatações acerca do comportamento da variação de cor da madeira. Em trabalho semelhante Costa et al, (2011) encontrou variação da cor igual a $\Delta \mathrm{E}^{*}=7,75$ e $\Delta \mathrm{E}^{*}=$ 6,43, para madeiras de jatobá e tauari, respectivamente, em tratamento com 4 ciclos totalizando 168 horas, as espécies foram classificadas como muito apreciável. Barreto e Pastore (2009) testaram a resistência ao intemperismo artificial de quatro madeiras tropicais em ciclos totalizando 2000 horas e encontraram valores de $\Delta \mathrm{E}^{*}=13,34$ para madeira de Itaúba, $\Delta \mathrm{E}^{*}=8,73$ para garapeira, e para marupá o valor foi de $\Delta \mathrm{E}^{*}=9,43$, apresentando variação de cor muito apreciável.

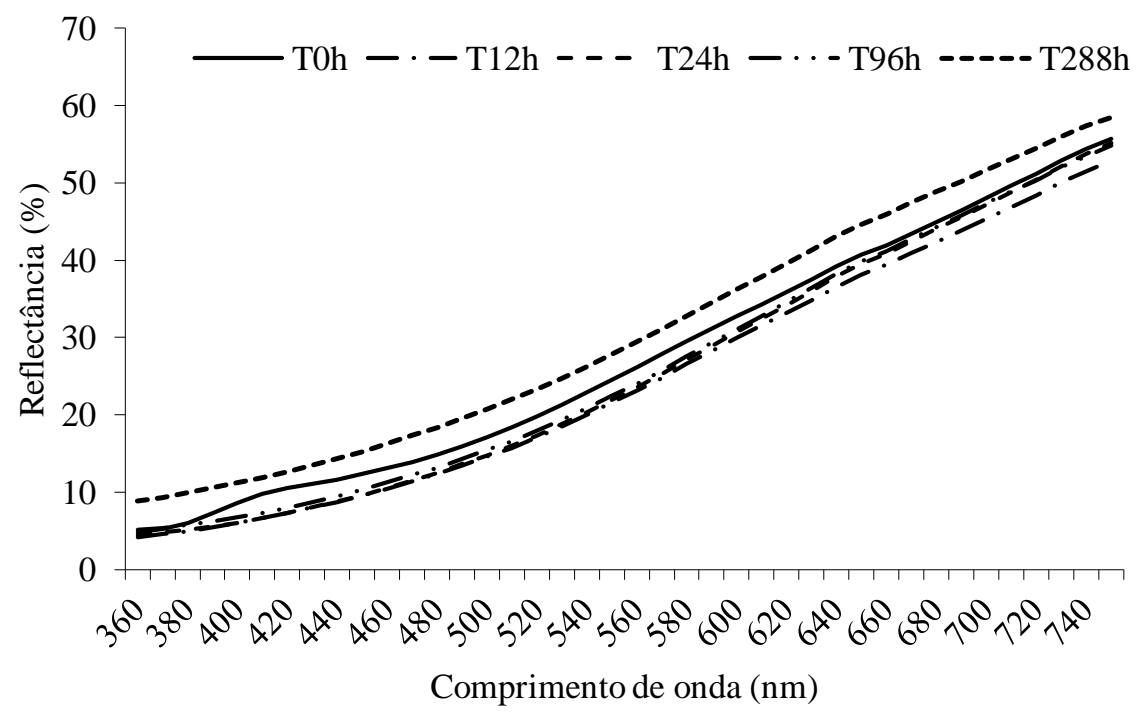

Figura 3. Reflectância das cores em função do comprimento de onda da madeira de Teca após $288 \mathrm{~h}$ de Radiação UV.

Figure 3. Reflectance of color depending on the wavelength of the teak wood after 288 hours of UV radiation.

FLORESTA, Curitiba, PR, v. 47, n. 1, p. 29 - 35, jan. / mar. 2017.

Mesquita, R. R. S. de et al.

ISSN eletrônico 1982-4688

DOI: $10.5380 /$ rf.v47i1.38562 
A figura 3 mostra o comportamento padrão das curvas de reflectância da madeira da espécie estudada. O percentual de reflectância caiu sensivelmente entre os tempos 0 e 12 horas, seguido de um aumento da reflectância entre os tempos 12 e 288 horas. O tratamento que apresentou maior reflectância foi o de 288 horas $(\mathrm{T} 288 \mathrm{H})$, seguido pela testemunha $(\mathrm{T} 0 \mathrm{H})$, é observado uma semelhança no comportamento das curvas antes do intemperismo (T0H) e após a ação do intemperismo (T288H).

\section{CONCLUSÕES}

- Os resultados encontrados no trabalho mostram que não houve variação significativa na cor natural da madeira de teca frente ao intemperismo para todos os tempos utilizados. Dessa forma, a cor original da madeira foi mantida e classificada como marrom oliva.

\section{REFERÊNCIAS}

AMERICAN SOCIETY FOR TESTING AND MATERIALS. ASTM D 2244 - Standard Practice for Calculation of Color Tolerances and Color Differences from Instrumentally Measured Color Coordinates. Philadelphia, USA, 2009.

AMERICAN SOCIETY FOR TESTING AND MATERIALS. ASTM G 154 - Standard Practice for Operating Fluorescent Light Apparatus for UV Exposure of Nonmetallic Materials. Philadelphia, PA, 2006.

AYADI, N.; LEJEUNE, F.; CHARRIER, F.; CHARRIER, B.; MERLIN, A. Color stability of heat-treated wood during artificial weathering. Holz als Roh und Werkstoff, v. 61, n. 3, p. 221-226, 2003.

BARRETO, C. C. K.; PASTORE, T. C. M. Resistência ao intemperismo artificial de quatro madeiras tropicais: o efeito dos extrativos. Revista Ciência Florestal, Santa Maria, v. 19, n. 1, p. 23-30, 2009.

BARROS, S. V. S.; MUNIZ, G. I. B.; MATOS, J. L. M. Caracterização colorimétrica das madeiras de três espécies florestais da Amazônia. Revista Cerne, v. 20, n. 3, p. 337-342, 2014.

CAMARgos, J. A. A.; GONÇALEZ, J. C. A colorimetria aplicada como instrumento na elaboração de uma tabela de cores de madeira. Brasil Florestal, Brasília, n. 71, p. 30-41, 2001.

COSTA, J. A.; GONÇALEZ, J. C.; CAMARGOS, J. A. A.; GOMES, I. A. S. Fotodegradação de duas espécies de madeiras tropicais: Jatobá (Hymenaea courbaril) e Tauari (Couratari oblongifolia) submetidas à radiação ultravioleta. Revista Cerne, Lavras, v. 17, n. 1, p. 133-139, 2011.

DA COSTA, R. B.; DE RESENDE, M. D. V. (2007). Experimentação e seleção no melhoramento genético de TECA (Tectona grandis Lf). Flor. Amb, v. 14, p. 76-92, 2007.

FEIST, W. C.; HON, D. N. S. Chemistry of weathering e protection. In: ROWELL, R. M. The chemistry of solid wood. Washington: Am. Chem. Soc, p. 614, 1984.

GONÇALEZ, J. C.; ZERBINI, N. J. Atividade florestal madeireira na área de influência do complexo hidrelétrico Belo Monte (PA): cadeia produtiva e propriedades tecnológicas de espécies pouco conhecidas. Brasília, UnB/ELETROBRAS ELETRONORTE/ANEEL/FINATEC, p. 128, 2010.

GONÇAlEZ, J. C.; FÉliX, T. L. F.; GOUVEIA, F. N.; CAMARGOS, J. A. A.; RIBEIRO, P. G; Efeito da radiação ultravioleta na cor da madeira de freijó (Cordia goeldiana Huber) após receber produtos de acabamentos. Revista Ciência Florestal, Santa Maria, v. 20, n. 4, p. 657-664, 2010.

HIKITA, Y.; TOYODA, T.; AZUMA, M. Weathering testing of timber: discoloration. In: IMAMURA, Y. High performance utilization of wood for outdooor uses. Kyoto: Press-Net, 2001.

HON, D. N. S. Weathering and photochemistry of wood. Wood and cellulosic chemistry. 2.ed. New York: Marcel Dekker, 2001.

MARTINS, S. A.; SANTOS, C. M. T.; GONÇALEZ, J. C.; CAMARGOS, J. A. A. Envelhecimento artificial acelerado por radiação ultravioleta de madeiras de Eucalyptus benthamii e Pinus caribaea var. hondurensis. Revista Floresta, v. 41, n. 1, 2011.

MIRANDA, I.; SOUZA, V.; PEREIRA, H. Wood properties of teak (Tectona grandis) from a mature unmanaged stand in East Timor. Journal of Wood Science, v. 57, n. 3, p. 171-178, 2011. 
MORI, C. L. S. O.; LIMA, J. T.; MORI, F. A.; TRUGILHO, P. F.; GONÇALEZ, J. C. Caracterização da cor da madeira de clones de híbridos de Eucalyptus spp. Revista Cerne, Lavras, v. 11, n. 2, p. 137-146, 2005.

MOYA, R.; PÉREZ, D. Processing and marketing of teakwood products from fast-grown teak plantations in Costa Rica. In: REGIONAL WORKSHOP PRODUCTS AND MARKETING OF TEAK WOOD PRODUCTS OF PLANTED FOREST. 2007. Proceedings...Peechi, India: KFRI, p. 24-32, 2007.

NZOKOU, P.; KAMDEM, D. P. Weathering of two hardwood species: African padauk (Pterocarpus soyauxii) and red maple (Acer rubrum). Journal of Tropical Forest Products, v. 8, n. 2, p. 200-209, 2002.

PANDEY, K. K.; PITMAN, A. J. FTIR. Studies of the changes in wood chemistry following decay by brown-rot and white-rot fungi.InternationalBiodeterioration\&Biodegradation. Birmingham, v. 52, n. 3, p. 151-160, 2002.

PANDEY, K. K. A note on the influence of extractives on the photo-discoloration and photo-degradation of wood. Polymer Degradation and Stability, v. 87, p. 375-379, 2005.

SILVA, J. O.; PASTORE, T. C. M.; PATORE JUNIOR, F. Resistência ao intemperismo artificial de cinco madeiras tropicais e de dois produtos de acabamento. Santa Maria. Revista Ciência Florestal, v. 17, n. 1, p. 1723, 2007.

TONINI, H.; COSTA, M. C. G.; SCWENGBER, L. A. M. Crescimento da teca (Tectona grandis) em reflorestamento na Amazônia Setentrional. Pesquisa Florestal Brasileira, n. 59, p. 05, 2010.

WILLIANS, R. S. Handbook of wood chemistry and composites. USDA, Forest Service, Forest Products, 171-178. 2005. 